\title{
Impact of some essential oils on the growth of toxigenic fungi and their toxin production
}

\author{
Zohri, A. A. ${ }^{1}$; Saber, S.M. ${ }^{2}$; Youssef, M.S. ${ }^{2}$ and Marwa Abdel-Kareem, ${ }^{2}$ \\ ${ }^{1}$ Botany \& Microbiology Department, Faculty of Science, Assiut University, Egypt \\ ${ }^{2}$ Botany Department, Faculty of Science, Sohag University, Egypt
}

Rec. 10 Jul, 2017 Accept. 12 Aug, 2017

\begin{abstract}
The impact of twelve essential oils (ginger, black pepper, black cumin, turmeric, baladi mint, peppery mint, cumin, marjoram lupine, cinnamon, thyme and cloves) on the growth of 11 toxigenic fungi and their ability for producing toxins were examined. Thyme, clove, baladi mint, peppery mint and cumin completely inhibited the growth of all tested fungi at two tested concentrations (10 and $50 \mu \mathrm{l} / 20 \mathrm{ml}$ medium). Marjoram essential oil completely inhibited the fungal growth at $50 \mu 1$. Cinnamon essential oil exhibited moderate inhibitory effect on the growth of all tested fungi at $50 \mu \mathrm{l}$. Ginger oil generally stimulated the growth of most the tested fungi. Black pepper, turmeric and lupine were recorded as low active oils. Whereas, Black cumin essential oil did not display any inhibitory effect on the growth of the toxigenic fungi at $50 \mu 1$. Thyme, clove and mint essential oils $(50 \mu \mathrm{l} / 50 \mathrm{ml}$ medium) completely inhibited toxin production by all the tested toxigenic fungi. Black pepper and ginger essential oils reduced mycotoxin formation.
\end{abstract}

Key words: toxigenic fungi, mycotoxins, essential oils.

\section{Introduction}

Spoilage and poisoning of foods by fungi is a major problem, especially in developing countries. Aspergillus, Fusarium and Penicillium species are the most important fungi causing spoilage of foodstuffs. Growth of these fugi in food crops are also responsible for off-flavour formation and production of allergenic compounds and mycotoxins, which lead to qualitative losses (Nielsen and Rios, 2000; Bennett and Klich, 2003). Aflatoxin $B_{1}$, Ochratoxin $\mathrm{A}$ and fumonisin $\mathrm{B}_{1}$ produced by these fungi display carcinogenic properties in humans and in laboratory animals, leading to the appearance of hepatocarcinoma (IARC, 1993; Pfohl-Leszkowicz and Manderville, 2007). However, in most countries, chemical and physical preservation are not permitted in foods. The need thus arises for natural preservatives that could be used for semi processed and processed foods. Currently the global trend is turned to safer and eco-friendly alternative approaches (Mari et al., 2007; Sharma et al., 2009). One of these possibilities is the use of essential oils to control mycotoxigenic fungi.

The antimicrobial properties of essential oils have been recognized and experimentally evaluated for many years. The essential oil have been involved in several applications such as natural antimicrobial agents in the field of pharmacology, phytopathology, clinical microbiology and food preservation. The essential oil preparations that possess antimicrobial activities have been the subject of many investigations resulted in screening of a wide variety of plant species, and have revealed structural and biological unique active compounds (Yoon et al., 1994; Vukovic et al., 2007).The general antifungal activity of essential oils is well documented (Alankararao et al., 1991; Gogoi et al., 1997; Meepagala et $a l ., 2002)$. The advantage of essential oils is their bioactivity in the vapour phase, a

* Corresponding author:
Dr. Marwa Abdel-Kareem
$凶$ marwaabdelkareem7@gmail.com


characteristic that makes them attractive as possible fumigants for stored product protection. Also, these essential oils are thought to play a role in plant defense mechanisms against phytopathogenic microorganisms (Mihaliak et al., 1991). Most of the essential oils have been reported to inhibit post harvest fungi in vitro conditions (Bellerbeck et al., 2001; Hidalgo et al., 2002).

Some of the essential oils have been reported to protect stored commodities from bio-deterioration. There are also some reports on essential oils in enhancing storage life of fruit and vegetables by controlling their fungal rotting. In this respect, Dubey and Kishore (1988) found that the essential oils from leaves of Melaleuca leucadendron, Ocimum canum and Citrus medica were able to protect several stored food commodities from biodeterioration caused by Aspergillus flavus and Aspergillus versicolor. The potential of using essential oils by spraying or dipping to control post harvest decay has been examined in fruits and vegetables (Tripathi and Dubey, 2004).

To control fungal contamination there are two possibilities, heat or chemical treatments, but it is necessary to replace chemical fungicides by natural products to avoid health problems. So, the present investigation aimed to evaluate the potential of 12 essential oils for bio-control of the fungal growth and toxin production by 11 toxigenic fungi.

\section{Materials and methods \\ Selection of toxigenic fungi}

A total of 11 toxigenic fungal isolates were selected for this study. Five highly toxigenic local isolates from different food sources in Sohag Governorate, Egypt named Aspergillus flavus 30 (Aflatoxin $\mathrm{B}_{1}, \mathrm{~B}_{2}, \mathrm{G}_{1}$ and $\mathrm{G}_{2}$ producer), A. ochraceus 76 (Ochratoxins A, B), Aspergillus nidulans 69 (Sterigmatocystin), Penicillium digitatum 131 (Patulin) and Alternaria alternata

5 (Alternariol). six highly toxigenic fungal isolates were purchased from CBS (Central Bureau voor Schimmelcultures), named Aspergillus parasiticus CBS 571.65 (Aflatoxin $\mathrm{B}_{1}, \mathrm{~B}_{2}, \mathrm{G}_{1}$ and $\mathrm{G}_{2}$ ), A. ochraceus CBS 589.68 (Ochratoxin A), Penicillium griseofulvum CBS 589.68 (Patulin), P. scabrosum CBS 530.97
(Fumagillin), Fusarium equiseti CBS 406.86 (Zearalenone) and Phaeosphaeria nodorum CBS 438.87 (Alternariol).

\section{Essential oils}

Essential oils of ginger, black pepper, black cumin, turmeric, baladi mint, peppery mint, cumin and marjoram were extracted using hot water steam distillation method (Kawther Abed, 2007). On the other hand, other four essential oils (lupine, cinnamon, thyme and cloves) were purchased from different markets at Sohag city.

The effect of essential oils on growth of toxigenic fungal isolates

The method described by Elena et al. (2009) was employed as follow: Fungi were grown in dishes on the potato dextrose agar medium. The oils were dripped on the covers of Petri dishes. Two different concentrations of each essential oil $(10$ and $50 \mu \mathrm{l})$ were tested after sowing the fungi and dripping oil. The dishes were sealed using the adhesive tape, turned over and put into a thermostat (temperature $28{ }^{\circ} \mathrm{C}$ ). The inhibition effect of the oil was detected by measuring the diameter of fungal colonies after 7 days of incubation and by comparing them to the control sample (without oil).

\section{The effect of essential oils on mycotoxins} production

To determine the effect of essential oils on mycotoxin formation, five oils were chosen as follow: baladi mint, thyme and cloves which completely inhibited all toxigenic fungal growth; black pepper which exhibited no antifungal activity and ginger which stimulated all toxigenic fungal growth. Each individual fungal isolate was cultivated on potato dextrose liquid medium. Erlenmeyer flasks of $250 \mathrm{ml}$ capacity were used. Each flask contained $50 \mathrm{ml}$ medium.

The flasks were sterilized at $121^{\circ} \mathrm{C}$ for 20 minutes and inoculated after cooling with two $\mathrm{ml}$ of the inoculum suspension of 10 days old culture of the pure organism. $50 \mu \mathrm{l}$ of tested essential oil were added. The cultures were incubated at $28 \pm 2^{\circ} \mathrm{C}$ as static cultivation for 10 days. At the end of incubation period, the content of each flask (medium + mycelium) were homogenized for five minutes in a high 
speed blender (16000 rpm) with $100 \mathrm{ml}$ chloroform. The extraction procedure was repeated three times. The combined chloroform extracts were washed with equal volume of distilled water, dried over anhydrous sodium sulphate, filtered then concentrated to near dryness. Mycotoxin levels were detected using thin layer chromatography (Scott et al., 1970; Gimeno, 1979; El-kady and Moubasher, 1982).

\section{Results and Discussion}

The antifungal activity of twelve essential oils on the growth of the eleven toxigenic fungal strains was examined and listed in tables $(1 \& 2)$. The results of the present study revealed that the essential oils at each of 10 and $50 \mu \mathrm{l} / 20 \mathrm{ml}$ of thyme, clove, mint baladi, peppery mint and cumin completely inhibited the growth of all tested toxigenic fungi. Marjoram essential oil at $50 \mu \mathrm{l} / 20 \mathrm{ml}$ medium (2500 ppm) completely inhibited the fungal growth and moderately inhibited them at 10 $\mu \mathrm{l} / 20 \mathrm{ml}$ medium (500 ppm).

The antifungal activity of thyme, clove and spearmint on the toxigenic fungi: A. flavus, $A$. parasiticus, A.ochraceus, A. fumigatus and Fusarium spp was demonstrated by MontesBelmont and Carvajall (1998) and Basilico and Basilico (1999). Montes-Belmont and Carvajall (1998) reported that the oils of clove and thymus caused a total inhibition of $A$. flavus on maize kernels. Nguefack et al. (2004) found that the essential oil of thymus inhibits the growth of various fungi involved in food spoilage, mycotoxin producers, pathogenic and wood decay fungi. Enas Amer (2012) examined the antifungal activity of six types of plant essential oil against the growth of 31 isolates of eight toxigenic fungal species and found that the oil of thyme completely suppressed the growth of all fungal isolates. Gorran et al. (2013) reported that thyme essential oil completely inhibits the growth of A. flavus at the concentration of $500 \mathrm{mg} / \mathrm{L}$. A concentration of 200-250 ppm of clove oil inhibited the growth of A. parasiticus (Bullerman et al., 1977).

Clove oil has also been found to be an effective inhibitor of Alternaria alternata, Fusarium oxysporum, $F$. culmorum, $F$. griseocyanus, Mucor circinelloides, Rhizopus stolonifer, Cladosporium cladosporioides, Penicillium citrinum, Saccharomyces cerevisiae and Aspergillus niger (Schmitz et al., 1993; Meena and Sethi, 1994). Soliman and Badeaa (2002) revealed that spearmint contained carfone as a main component of its essential oil, which may be responsible for their antifungal activity.

Cinnamon essential oil at $50 \mu \mathrm{l} / 20 \mathrm{ml}$ medium moderately inhibited the growth of all tested toxigenic fungi (Tables $1 \& 2$ ). Sukatta et al. (2008) previously showed that mixing clove and cinnamon oils at the appropriate ratios result in an improvement of the efficacy against the post harvest decay fungi of grapes. Also cinnam on oil is a potential inhibitor of Penicillium expansum which is a cause of spoilage of apples (Ryu and Holt, 1993).

Ginger oil generally stimulated the growth of most toxigenic fungi under study except $A$. flavus 30 and Penicillium griseofulvum CBS 589.68 at 50 and $10 \mu 1 / 20 \mathrm{ml}$ medium (Tables, $1 \& 2$ ). Similar results were observed by Mabrouk and EL-Shayeb (1981) who reported that ginger stimulated fungal growth at all the concentration tested. Black cumin essential oil displayed no antifungal effect on the growth at both tested concentrations. Similar findings were reported by Maraqa et al. (2007) using black cumin essential oil.

Other three oils named black pepper, turmeric and lupine were recorded as low active oils at $50 \mu \mathrm{l} / 20 \mathrm{ml}$ medium and had no inhibitory effect on the growth of the different toxigenic fungal strains using $10 \mu \mathrm{l} / 20 \mathrm{ml}$ medium(Tables, $1 \&$ 2). In contrast, Sindhu et al. (2011) evaluated the potential of turmeric on control of A. flavus growth and aflatoxin production. Bokhari (2007) reported that black pepper did not affect the growth of the toxigenic $A$. versicolor.

The results in tables $3 \& 4$ indicated the important role of essential oils (especially thyme, clove and mint) in inhibiting toxin production by all the 11 toxigenic fungi under examination. The inhibitory effects of some plants essential oils against aflatoxin biosynthesis by $A$. flavus and A. parasiticus were reported in previous studies (El- Kady et al., 2000; Attanda et al., 2007; Mohamed et al., 2011). The effects of clove essential oil on 
growth and mycotoxin production by some toxigenic fungal genera such as Aspergillus spp., Penicillium spp., and Fusarium spp. have been reported (Velluti et al., 2004; Nesci et al., 2005, 2011). Patkar et al. (1993) reported that clove essential oil inhibited either aflatoxin or ochratoxin accumulation in different substrates. Reddy et al. (2010) reported the efficacy of certain plant extracts on mycelial growth of $A$. ochraceus and ochratoxin biosynthesis. The oils of thyme and cinnamon completely inhibit all the test fungi and ochratoxin production at $3000 \mathrm{ppm}$ (Soliman and Badeaa, 2002). Velluti et al. (2004) found that clove essential oil was able to inhibit zearalenone and deoxynevalenol synthesis under certain environmental conditions in sterile maize inoculated with Fusarium species.

In this study black pepper essential oil completely inhibited toxin production of each of $F$. equiseti CBS 406.86, P. nodorum CBS 438.87, A. alternata 5 and A. nidulans 69. Also, it reduced fumagillin production by $P$. scabrosum CBS 530.97 and Patulin production by $P$. digitatum 131 to 40 and $20 \%$, respectively. Black pepper essential oil did not inhibit the production of aflatoxin and ochratoxin using both standard and local fungal strains. Ito et al. (1994) reported that pepper extracts have the ability to reduce aflatoxin production in $A$. parasiticus IFO 30179 and A. flavus var. columnaris S46.

Ginger oil completely inhibited the production of zearalenone and alternariol and reduced the patulin formed by standard and local fungal strains to 40 and $80 \%$, respectively. Fumagillin production was reduced by $20 \%$ using ginger essential oil, while the production of sterigmatocystin was stimulated by $20 \%$ using this oil. Aflatoxin and ochratoxin production by the standard and local strains were not affected by the presence of ginger essential oil. Ginger has been listed in Generally Recognized as Safe (GRAS) and has antimicrobial and antimycotoxigenic ef fects (Tatsadjieu et al., 2009) and also because of its aroma and taste, it has been used for culinary purposes from ages. Ginger essential oil is indeed effective against several mycotoxins in stored commodities (Sharma et al., 2013).

\begin{tabular}{|c|c|c|c|c|c|c|c|c|c|c|c|c|c|c|}
\hline \multirow{2}{*}{\multicolumn{2}{|c|}{ Essential oils }} & \multirow{2}{*}{$\begin{array}{l}\text { Conc. of } \\
\text { essentialol } \\
(\mu / 20 \mathrm{ml} \\
\text { medimm }\end{array}$} & \multicolumn{2}{|c|}{$\begin{array}{l}\text { A parapitigur } \\
\text { CBS 571.65 }\end{array}$} & \multicolumn{2}{|c|}{$\begin{array}{l}\text { A echlugecens } \\
\text { CBS 588.68 }\end{array}$} & \multicolumn{2}{|c|}{ 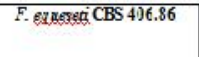 } & \multicolumn{2}{|c|}{$\begin{array}{l}\text { P. gatiegefu'vem, CBS } \\
\text { 31E.63 }\end{array}$} & \multicolumn{2}{|c|}{ 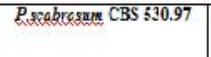 } & \multicolumn{2}{|c|}{$\begin{array}{l}\text { Phageropogeric agderum } \\
\text { CBS } 433.87\end{array}$} \\
\hline & & & $\begin{array}{c}\text { Fangal } \\
\text { grout }(\mathrm{cm})\end{array}$ & Ishibiian 96 & $\begin{array}{c}\text { Fungal } \\
\text { gronth (cm) }\end{array}$ & Inhib:tion 96 & $\begin{array}{c}\text { Fungal } \\
\text { grouth (cm) }\end{array}$ & Intibition 96 & $\begin{array}{c}\text { Fungal } \\
\text { grouth (m) }\end{array}$ & Inb bition 96 & $\begin{array}{c}\text { Fangal } \\
\text { grovt (cm) }\end{array}$ & Inbibiicn qu $^{2}$ & $\begin{array}{c}\text { Fungal } \\
\text { grounth (cm) }\end{array}$ & Inh.bition 96 \\
\hline \multirow{13}{*}{$\begin{array}{l}\text { Extracted } \\
\text { essential } \\
\text { oils }\end{array}$} & contrcl & 0 & 7.0 & 0 & t. 0 & 0 & 7.0 & 0 & $\hat{\lambda .5}$ & 0 & 20 & 0 & 3.0 & 0 \\
\hline & \multirow{2}{*}{$\begin{array}{l}\text { Black } \\
\text { cumin }\end{array}$} & 10 & 70 & c & 5.0 & 0 & 7.0 & 0 & 2.5 & 0 & 20 & c & 3.0 & 0 \\
\hline & & 50 & 7.0 & $c$ & 5.0 & 0 & 7.0 & 0 & 2.5 & 0 & 20 & $c$ & 3.0 & 0 \\
\hline & \multirow{2}{*}{\begin{tabular}{|l} 
Black \\
pepper
\end{tabular}} & 10 & 7.0 & $c$ & 5.0 & 0 & 7.0 & 0 & 2.5 & 0 & 20 & c & 3.0 & 0 \\
\hline & & 50 & 6.8 & 3 & 4.3 & 14 & 6.5 & 7 & 2.5 & 0 & 15 & 25 & 2.5 & 17 \\
\hline & \multirow[t]{2}{*}{ Cunir } & 10 & 0.8 & 89 & 1.0 & 80 & . & 100 & - & 100 & . & 100 & - & 100 \\
\hline & & 50 & . & 100 & - & 100 & . & 100 & - & 100 & . & 100 & - & 100 \\
\hline & \multirow[t]{2}{*}{ Ginger } & 10 & 72 & +2 & 5.3 & +6 & 7.5 & +7 & 2.5 & 0 & 22 & +10 & 3.2 & +6 \\
\hline & & 50 & 74 & +5 & 5.5 & +10 & 7.8 & +11 & 2.5 & 0 & 2.4 & +20 & 3.2 & +6 \\
\hline & \multirow[t]{2}{*}{ Marioran } & 10 & 65 & 7 & 2.8 & 44 & 5.4 & 23 & 1.8 & 28 & 14 & 30 & - & 100 \\
\hline & & 50 & . & 100 & - & 100 & . & 100 & . & 100 & . & 100 & - & 100 \\
\hline & \multirow[t]{2}{*}{$\begin{array}{l}\begin{array}{l}\text { Balagdi } \\
\text { miat }\end{array} \\
\text { mat }\end{array}$} & 10 & 5.8 & 17 & 2.0 & 60 & 2.5 & 65 & 1.2 & 52 & 0.8 & 60 & - & 100 \\
\hline & & 50 & . & 100 & - & 100 & . & 100 & - & 100 & . & 100 & . & 100 \\
\hline
\end{tabular}

Table (1): Impact of some extracted and purchased essential oils (10 \&50 $\mu \mathrm{l} / 20 \mathrm{ml}$ medium) on the growth of standard toxigenic fungi. 


\begin{tabular}{|c|c|c|c|c|c|c|c|c|c|c|c|c|c|c|}
\hline \multirow{2}{*}{\multicolumn{2}{|c|}{ Essential oils }} & \multirow{2}{*}{$\begin{array}{l}\text { Conc, of essential } \\
\text { oil ( }(\mu / 20 \mathrm{ml} \\
\text { mediun) }\end{array}$} & \multicolumn{2}{|c|}{$\begin{array}{l}\text { A. pugarificus } \\
\text { CBS } 571.65\end{array}$} & \multicolumn{2}{|c|}{$\begin{array}{l}\text { A edkrscens } \\
\text { CBS } 589.68\end{array}$} & \multicolumn{2}{|c|}{ F. equzsed CBS 406.86} & \multicolumn{2}{|c|}{$\begin{array}{l}\text { P. giseofflyum CBS } \\
\text { 315.63 }\end{array}$} & \multicolumn{2}{|c|}{ Prscelorezum CBS \$30.97 } & \multicolumn{2}{|c|}{ 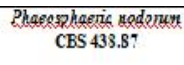 } \\
\hline & & & $\begin{array}{c}\text { Fungal } \\
\text { grounth (m) } \\
\end{array}$ & $\begin{array}{c}\text { Inhbibition } \\
96\end{array}$ & $\begin{array}{c}\text { Fuagal grouth } \\
\text { (cm) }\end{array}$ & \begin{tabular}{|c} 
Isbibition \\
$\%$ \\
\end{tabular} & $\begin{array}{c}\text { Fargalgronth } \\
\text { (cm) } \\
\end{array}$ & $\begin{array}{c}\text { Irhibitos } \\
04 \\
\end{array}$ & $\begin{array}{c}\text { Furgal grvith } \\
\text { (m) }\end{array}$ & $\begin{array}{c}\text { Inbijition } \\
c_{0}\end{array}$ & $\begin{array}{c}\text { Fungal } \\
\text { grouta (cni) }\end{array}$ & $\begin{array}{c}\text { Inbibiton } \\
9 ; \\
\end{array}$ & $\begin{array}{c}\text { Fungal } \\
\text { grounth (m) }\end{array}$ & $\begin{array}{c}\text { Insibition } \\
36 \\
\end{array}$ \\
\hline \multirow{5}{*}{$\begin{array}{l}\text { Extracted } \\
\text { essential oils }\end{array}$} & cortrol & 0 & 7.0 & 0 & 5.0 & 0 & 7.0 & 0 & 2.5 & 0 & 2.0 & 0 & 3.0 & 0 \\
\hline & $\begin{array}{l}\text { Peppery } \\
\text { nint }\end{array}$ & 10 & 5.8 & 17 & - & 100 & - & 100 & 1.5 & 40 & 0.7 & 65 & - & 100 \\
\hline & & 50 & - & 100 & - & 100 & . & 160 & - & 100 & . & 160 & - & 100 \\
\hline & Turmeric & 10 & 7.0 & 0 & 5.0 & 0 & 7.0 & $\mathrm{c}$ & 2.5 & 0 & 2.0 & $\mathrm{C}$ & 3.0 & 0 \\
\hline & & 50 & 6.8 & 3 & 3.5 & 30 & 6.3 & 10 & 1.8 & 28 & 18 & 10 & $\therefore .0$ & 67 \\
\hline \multirow{8}{*}{$\begin{array}{l}\text { Purchased } \\
\text { essential oils }\end{array}$} & Cirnamon & 10 & 7.0 & 0 & 5.0 & 0 & 7.0 & c & 2.5 & 0 & 20 & 0 & 3.0 & 0 \\
\hline & & 50 & 5.5 & $2:$ & 3.5 & 30 & 4.0 & 43 & 2.2 & 12 & 0.6 & 70 & $\therefore .0$ & 67 \\
\hline & Cleves & 10 & - & 100 & - & 100 & . & 100 & - & 100 & . & 160 & - & 100 \\
\hline & & 50 & - & 100 & - & 100 & . & 100 & - & 100 & . & 100 & - & 100 \\
\hline & Lupine & 10 & 7.0 & 0 & 5.0 & 0 & 7.0 & $\mathrm{c}$ & 2.5 & 0 & 20 & $c$ & 3.0 & 0 \\
\hline & & 50 & 6.2 & 1: & 4.6 & 8 & 6.5 & 7 & 1.0 & 60 & 12 & 4) & .3 & 57 \\
\hline & Thyme & 10 & - & 100 & - & 100 & - & 100 & - & 100 &. & 100 & - & 100 \\
\hline & & 50 & - & 100 & - & 100 & . & 100 & - & 100 & - & 160 & - & 100 \\
\hline
\end{tabular}

Table (1): Continued

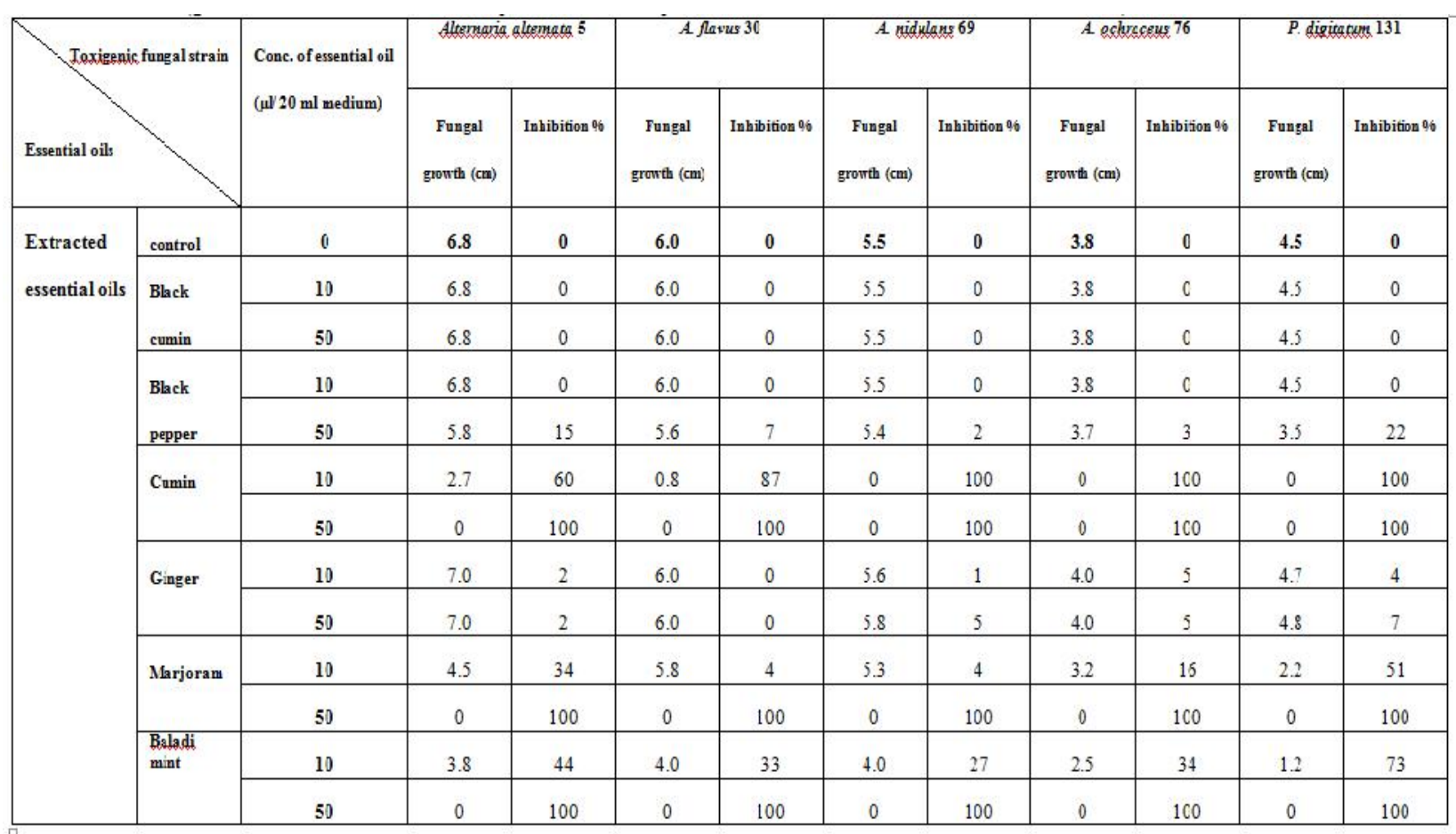

Table (2): Impact of some extracted and purchased essential oils ( $10 \& 50 \mu \mathrm{l} / 20 \mathrm{ml}$ medium $)$ on the growth of local toxigenic fungi (growth diameter measured by $\mathrm{cm}$ after 7 days of incubation on PDA medium at $28^{\circ} \mathrm{C}$ ). 


\begin{tabular}{|c|c|c|c|c|c|c|c|c|c|c|c|c|}
\hline \multirow{2}{*}{\multicolumn{2}{|c|}{ Essertial cils }} & \multirow{3}{*}{$\begin{array}{l}\text { Corc. of essential oil } \\
\text { (al/ } 20 \mathrm{ml} \text { mediun) } \\
0\end{array}$} & \multicolumn{2}{|c|}{ 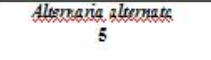 } & \multicolumn{2}{|c|}{$\begin{array}{l}\text { A. Ravus } \\
\mathbf{3 0}\end{array}$} & \multicolumn{2}{|c|}{ A ridulong 69} & \multicolumn{2}{|c|}{$\begin{array}{l}\text { A oclugasu: } \\
76\end{array}$} & \multicolumn{2}{|c|}{ P. disitatum 131} \\
\hline & & & $\begin{array}{c}\text { Fungal } \\
\text { gronth (cm) }\end{array}$ & Inbibition 96 & $\begin{array}{l}\text { Fungal } \\
\text { gronth (cm) }\end{array}$ & In & $\begin{array}{c}\text { Furgal } \\
\text { growth (cm) }\end{array}$ & Inbibition 16 & $\begin{array}{c}\text { Fungal } \\
\text { growta (cm) }\end{array}$ & Inhijition 96 & $\begin{array}{l}\text { Fusgel } \\
\text { growth (cmi) }\end{array}$ & Inhibifion 96 \\
\hline \multirow{5}{*}{$\begin{array}{l}\text { Extracted } \\
\text { essentialoils }\end{array}$} & control & & 6.8 & 0 & 6.9 & 0 & 5.5 & 0 & 3.8 & 0 & 4.5 & 0 \\
\hline & \multirow[t]{2}{*}{$\begin{array}{l}\text { Peppery } \\
\text { mint }\end{array}$} & 10 & 4.2 & 38 & 4.5 & 25 & 4.3 & 22 & 1.5 & 61 & 1.2 & 73 \\
\hline & & 50 & 0 & 100 & 0 & $10 \mathrm{C}$ & 0 & 100 & 0 & .00 & 0 & 100 \\
\hline & \multirow[t]{2}{*}{ Turmeric } & 10 & 6.8 & 0 & 6.0 & 0 & 5.5 & 0 & 38 & 0 & 4.5 & 0 \\
\hline & & 50 & 5.0 & 27 & 5.3 & 3 & 5.4 & 2 & 3.5 & 8 & 3.6 & 20 \\
\hline \multirow{8}{*}{$\begin{array}{l}\text { Purchased } \\
\text { essentialoils }\end{array}$} & \multirow[t]{2}{*}{ Cinnamon } & 10 & 6.8 & 0 & 6.0 & 0 & 5.5 & 0 & 3.8 & 0 & 4.5 & 0 \\
\hline & & 50 & 6.2 & 9 & 5.3 & 3 & 3.5 & 37 & 36 & 5 & 3.5 & 22 \\
\hline & \multirow[t]{2}{*}{ Cloves } & 10 & 0 & 100 & 1.2 & 80 & 2.8 & 49 & 0 & .00 & 0 & 100 \\
\hline & & 50 & 0 & 100 & 0 & $10 \mathrm{C}$ & 0 & 100 & 0 & .00 & 0 & 100 \\
\hline & \multirow[t]{2}{*}{\begin{tabular}{|l} 
Lupire \\
\end{tabular}} & 10 & 6.8 & 0 & 6.0 & 0 & 5.5 & 0 & 3.8 & 0 & 4.5 & 0 \\
\hline & & 50 & 5.8 & 15 & 5.7 & 5 & 5.4 & 2 & 33 & 13 & 3.2 & 29 \\
\hline & \multirow[t]{2}{*}{ Thywe } & 10 & 0 & 100 & 0 & $10 \mathrm{C}$ & 0 & 100 & 0 & .00 & 0 & 100 \\
\hline & & 50 & 0 & 100 & 0 & $10 \mathrm{C}$ & 0 & 100 & 0 & .00 & 0 & 100 \\
\hline
\end{tabular}

Table (2): Continued

\begin{tabular}{|c|c|c|c|c|c|c|c|c|c|c|c|c|c|}
\hline \multicolumn{2}{|c|}{ Iorigenic fungal strain } & \multicolumn{2}{|c|}{$\begin{array}{l}\text { A. potositiculs } \\
\text { CBS 57l.65 }\end{array}$} & \multicolumn{2}{|c|}{$\begin{array}{l}\text { A. ochroceus } \\
\text { CBS } 589.68\end{array}$} & \multicolumn{2}{|c|}{$\begin{array}{l}\text { F. equesetic CBS } \\
\text { 406.86 }\end{array}$} & \multicolumn{2}{|c|}{$\begin{array}{l}\text { P. griseffulumm } \\
\text { CBS } 315.63\end{array}$} & \multicolumn{2}{|c|}{$\begin{array}{l}\text { P. scabbesiun CBS } \\
530.97\end{array}$} & \multicolumn{2}{|c|}{$\begin{array}{c}\text { Phueasphoeria } \\
\text { modorum CBS } 438.87\end{array}$} \\
\hline Essential oils & & $\begin{array}{l}\text { Visual } \\
\text { grovth }\end{array}$ & $\begin{array}{c}\text { Llibibition of } \\
\text { torin } \\
\text { production } \\
\%\end{array}$ & $\begin{array}{l}\text { Vistal } \\
\text { grenth }\end{array}$ & $\begin{array}{l}\text { Inlibition } \\
\text { of torin } \\
\text { production } \\
\%\end{array}$ & $\begin{array}{l}\text { Tisual } \\
\text { grouth }\end{array}$ & $\begin{array}{c}\text { Lrubitition } \\
\text { eftoxir } \\
\text { prodtction } \\
\%\end{array}$ & $\begin{array}{l}\text { Visual } \\
\text { grvorth }\end{array}$ & $\begin{array}{l}\text { Inhisition } \\
\text { of forin } \\
\text { preduction } \\
\%\end{array}$ & $\begin{array}{l}\text { Tisual } \\
\text { gromth) }\end{array}$ & $\begin{array}{l}\text { Irulibition } \\
\text { ef foxir } \\
\text { prodrctior } \\
\%\end{array}$ & $\begin{array}{l}\text { Tisual } \\
\text { gromith }\end{array}$ & $\begin{array}{l}\text { Inibitition of } \\
\text { toxin } \\
\text { production } \\
\%\end{array}$ \\
\hline \multirow{4}{*}{$\begin{array}{l}\text { Extracted } \\
\text { essential ols }\end{array}$} & Control & 5 & 0 & 4 & 0 & 5 & 0 & 5 & 0 & 5 & 0 & 4 & 0 \\
\hline & $\begin{array}{l}\text { Black } \\
\text { pepper }\end{array}$ & 4 & 0 & 3 & 0 & 3 & .00 & 4 & -20 & 4 & 6) & 2 & 100 \\
\hline & Ginger & 5 & 0 & 4 & 0 & j & $: 00$ & 5 & 60 & j & 20 & 5 & 100 \\
\hline & Mint & . & 100 & . & 100 & . & .00 & . & 100 & . & $: 00$ & . & 100 \\
\hline \multirow{2}{*}{$\begin{array}{l}\text { Purchased } \\
\text { essential oils }\end{array}$} & Cloves & - & 100 & - & 100 & . & $: 00$ & - & 100 & . & $: 00$ & - & 100 \\
\hline & Thrme & - & 100 & - & 100 & . & .00 & - & 100 & . & $: 00$ & - & 100 \\
\hline
\end{tabular}

Table (3): Impact of some extracted and purchased essential oils (50 $\mu 1 / 50 \mathrm{ml}$ medium) on fungal growth and mycotoxins formation by some standard toxigenic fungal strains grown on PDA liquid medium supplemented with the essential oil at $28^{\circ} \mathrm{C}$ for 10 days. 


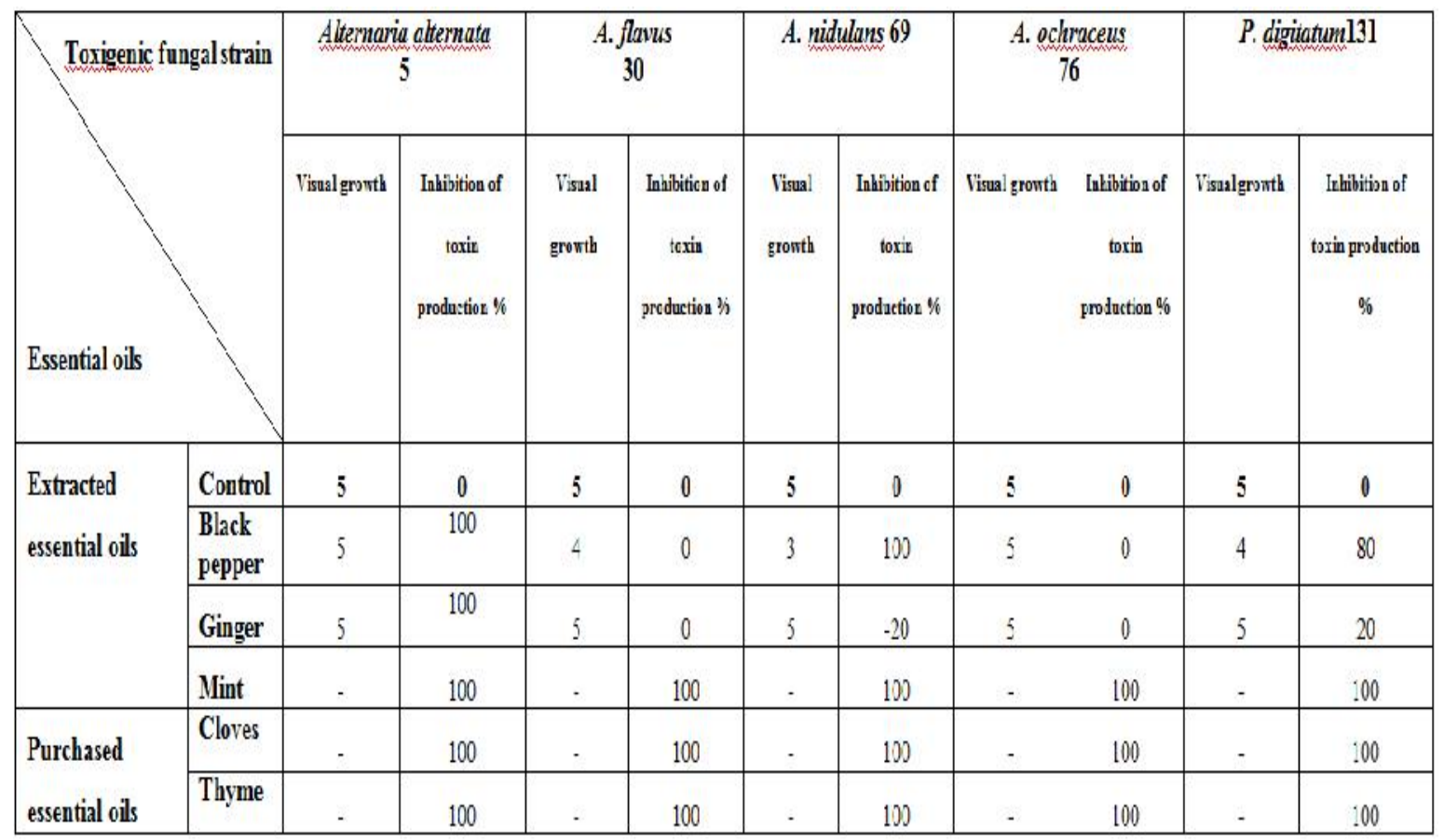

Table (4): The inhibitory effect (\%) of some extracted and purchased essential oils $(50 \mu \mathrm{l} / 50 \mathrm{ml}$ medium) on fungal growth and mycotoxins formation by some local toxigenic fungal strains grown on PDA liquid medium supplemented with the essential oil at $28^{\circ} \mathrm{C}$ for 10 days.

\section{References:}

Alankararao, GSJG, Baby, P., Rajendra Prasad Y. (1991). Leaf oil of Coleus amboinicus Lour.: the in vitro antimicrobial studies. Perfumerie Kosmetics 72: 744-745.

Attanda, O.O., Akpan, I., Oluwafemi, F. (2007). The potential of some spice essential oils in the control of $A$. parasiticus CFR 223 and aflatoxin production. Food Control. 18: 601607.

Basilico, M.Z., Basilico, J.C. (1999). Inhibitory effect of some spice essential oils on Aspergillus ochraceus NRRL 3174 growth and ochratoxin production. Appl. Microbiol. 29 (4): 238-241.

Bellerbeck, V.G., De Roques, C.G., Bessiere, J.M., Fonvieille, J.L., Dargent, R. (2001). Effect of Cymbopogon nardus (L.) W. Watson essential oil on the growth and morphogenesis of Aspergillus niger.Can. J. Microbiol. 47: 9-17.
Bennett, J.W., Klich, M. (2003). Mycotoxins. Clin. Microbiol. Rev. 16: 497-516.

Bokhari, F.M. (2007). Spices mycobiota and mycotoxins available in Saudi Arabia and their abilities to inhibit growth of some toxigenic fungi. Mycobiol. 35(2): 47-53.

Bullerman, L.B., Lieu, F.Y., Seier, A.S. (1977). Inhibition of growth and aflatoxin production by cinnamon and clove oils, cinamic aldehyde and eugenol. J. Food Sci. 42: 1107-1108.

Dubey, N.K., Kishore, N. (1988). Exploitation of higher plant products as natural fumigants. In: Proceedings of the Fifth International Congress on Plant Pathology, Kyoto, Japan, p. 423.

Elena, P.C., Rocio, J., Julio, E.P., Manuel, A., Javier, V. (2009). Antioxidant activity of seed polyphenols in fifteen wild Lathyrus species from South Spain., Food Sci. Technol. 42: 705-709.

El-Kady, I.A., Moubasher, M.H. (1982). Toxigenicity and toxin of 
Stachybotrys chartarum isolates from wheat straw samples in Egypt. Exp. Mycol. 6: .25 - 31 .

El-Kady, I.A., El-Maraghy, S.S., Zohri, A.A. (2000). Effect of some spices and essential oils on the growth and aflatoxin production by Aspergillus flavus IMI 89717 on liquid medium and minced meat. The First Symposium on Food Safety, The Proceeding of College of Agric. \& Food Sci. King Faisal Univ. (K. S. A. ): 215-224.

Enas Amer, M. (2012). Study on potential mycotoxigenic fungal contaminants isolated from operation rooms and intensive care units of Assiut University Hospitals. MSc Thesis, Department of Botany, Faculty of Science, Sohag University, Sohag, Egypt.

Gimeno, A. (1979). Thin-layer chromatographic determination of aflatoxins, ochratoxins, sterigmatocystin, zearalenone, citrinin, T-2 toxin, diacetoxyscirpenol, penicillic acid and penitrem "A". J. Assoc. Off. Anal. Chem. 62: 579- 585.

Gogoi, R., Baruah P, Nath SC (1997). Antifungal activity of the essential oil of Litsea cubeba Pers. J. Essential Oils Res. 9:213-215.

Gorran, A., Farzaneh, M., Shivazad, M., Rezaeian, M., Ghassempou, A. (2013). Aflatoxin $B_{1}$-reduction of Aspergillus flavus by three medicinal plants (Lamiaceae). Food Control. 31: 218-223.

Hidalgo, P.J., Ubera, J.L., Santos, J.A., LaFont, F., Castelanos, C., Palomino, A., Roman, M. (2002). Essential oils in Culamintha sylvatica. Bromf. ascendens (Jorden) P.W. Ball wild and cultivated productions and antifungal activity. J. Essential Oil Res. 14: 6871.

IARC (1993). Some naturally occurring substances, food items and constituents, heterocyclic aromatic amines and mycotoxins, in Monographs on the evaluation of carcinogenic risks to humans. World health organization, Lyon.

Ito, H., Chen, H., Bunnak, J. (1994). Aflatoxin production by microorganisms of the Aspergillus flavus group in spices and the effect of irradiation. J. Sci. Food Agric. 65: 141-142.

Kawther, F., Abed (2007). Antimicrobial activity of essential oils of some medicinal plants from Saudi Arabia. Saud. J. Biol. Sci. 14 (1): 53-60.

Mabrouk, S.S., EL-Shayeb, M.M.A. (1981). Inhibition of aflatoxin formation by some spices. Z. Lebesm-Unters. Forsch. 71: 344-347.

Maraqa, A., Alsharoa, N.F., Farah, H., Albjeirami, W.M., Shakya, A.K., Sallal, A.J. (2007). Effect of Nigella sativa extract and oil on aflatoxin production by Aspergillus flavus. Turkish J. Biol. 31: 155-159.

Mari, M., Neri, F., Bertolini, P. (2007). Novel approaches to prevent and control postharvest diseases of fruit. Stewart Postharvest Review, Redhill. 3:1-7.

Meena, M.R., Sethi, V. (1994). Antimicrobial activity of essential oils from spices. J. Food Sci. Technol. 31: 68-70.

Meepagala, K.M., Sturtz, G., Wedge, D.E. (2002). Antifungal constituents of the essential oil fraction of Artemisia drancunculus L. var. dracunculus. J. Agric. Food Chem. 50:6989-6992.

Mihaliak, C.A., Gershenzo, J., Croteau, R, (1991). Lack of rapidmonoterpene turnover in rooted plants, implications for theories of plant chemical defense. Oecologia 87: 373-376.

Mohamed, M.D., Neveen, HAE-S, Lamia TAE-K (2011): In vitro inhibition of growth and aflatoxin $\mathrm{B} 1$ production of Aspergillus flavus strain (ATCC 16872) by various medicinal plant essential oils. Macedonian J. Med. Sci.4 (4): 345-350.

Montes-Belmont, R., Carvajal, M. (1998). Control of Aspergillus flavus in maize with plant essential oils and 
their components.J. Food Prot. 61(5): 616-619.

Nesci, A.V., Bluma, R.V., Etcheverry, M.G. (2005). In vitro selection of maize rhizobacteria to study potential biological control of Aspergillus section Flavi and aflatoxin production. E. J. Plant Pathol. 113 (2):159-171.

Nesci, A., Montemarani, A., Passone, M.A., Etcheverry, M. (2011). Insecticidal activity of synthetic antioxidants, natural phytochemicals, and essential oils against an Aspergillus section Flavi vector (Oryzaephilus surinamensis L.) in microcosm. J. Pest Sci. 84: 107-115.

Nguefack, J., Leth, V., Zollo, A., Mathur, S.B. (2004). Evaluation of five essential oils from aromatic plants of Cameroon for controlling food spoilage and mycotoxin producing fungi. Int. J. Food Microbiol. 94: 329-334.

Nielsen, P.V., Rios, R. (2000). Inhibition of fungal growth on bread by volatile components from spices and herbs, and the possible application in active packaging, with special emphasis on mustard essential oil. Int. J. Food Microbiol. 60: 219-229.

Patkar, K., Usha, C., Shetty, H., Poster, N., Lacey J (1993). Effect of spice essential oils on growth and aflatoxin $\mathrm{B}_{1}$ production by $A$. flavus. Letter in Appl. Microbiol. 17(2): 49-51.

Pfohl-Leszkowicz, A., Manderville, R.A. (2007). Ochratoxin A toxicity: an overview on toxicity and carcinogenicity in animals and humans. Mol. Nut. Food Res. 51:6169.

Reddy, K.R.N., Raghavender, C.R., Reddy, B.N., Salleh, B. (2010). Biological control of Aspergillus flavus growth and subsequent aflatoxin $B_{1}$ production in sorghum grains. Afri. J. Biotechnol. 9(27): 4247-4250.

Ryu D, Holt DL (1993). Growth inhibition of Penicillium expansum by several commonly used food ingredients, J. Food Prot. 56: 862-867.

Schmitz, S., Weidenbgner, M., Kunaz, B. (1993). Herbs and spices as selective inhibitors of mould growth, Chemie, Mikrobiologie Technologie der Lebensmitte 15: 175-177.

Scott, P.M., Lawrence, J.W., Van Walbeak, W. (1970). Detection of mycotoxins by thin-layer chromatography: Application to screening of fungal extracts. Appl. Microbiol. 20: 839 842.

Sharma, R.R., Singh, D., Singh, R. (2009). Biological control of postharvest diseases of fruits and vegetables by microbial antagonists: A review. Biol. Control. 50(3): 205-221.

Sindhu, S., Chempakam, B., Leela, N.K., Suseela Bhai, R. (2011). Chemoprevention by essential oil of turmeric leaves (Curcuma longa L.) on the growth of Aspergillus flavus and aflatoxin production. Food Chem. Toxicol. 49: 1188-1192.

Solimana, K.M., Badeaa, R.I. (2002). Effect of oil extracted from some medicinal plants on different mycotoxigenic fungi. Food Chem. Toxicol. 40: 1669-1675.

Sukata, V., Haruthaithanosan, V., Chntarapanont W, Dilokkunanat V, Suppakul, P. (2008). Antifungal activity of clove and cinnamon oil and their synergistic against postharvest decay fungi of grape in vitro. Nat. Sci. 42:169-174.

Tatsadjieu, N.L., Dongmo, P.M.J., Ngssoum, M.B., Etoa, F.X., Mbofung, C.M.F. (2009). Investigation on the essential oil of lippiarugosa from Cameroon for its potential use as antifungal agent against Aspergillus flavus Link E.X. Fries. Food Control 20: 161166.

Tripathi P, Dubey NK (2004). Exploitation of natural products as an alternative strategy to control postharvest fungal rotting of fruit and vegetables. Post. Biol and Technol. 32: 235-245. 
Velluti, A., Sanchis, V., Ramos, A.J., Turon, C., Marin, S. (2004). Impact of essential oils on growth rate, zearalenone and deoxynivalenol production by Fusarium graminearum under different temperature and water activity conditions in maize grain. J. Appl. Microbiol. 96:716-724.
Vukovic, N., Milosevic, T., Sukdolak, S., Solujic, S. (2007). Antimicrobial activities of essential oil and methanol extract of Teucrium montanum. 4:17-20.

Yoon, S.Y., Eo SK, Lee DK, Han, S.S. (1994). Antimicrobial activity of Ganoderma lucidum extract alone and in combination with some antibiotics, Arch. Pharm. Res. 6: 4.

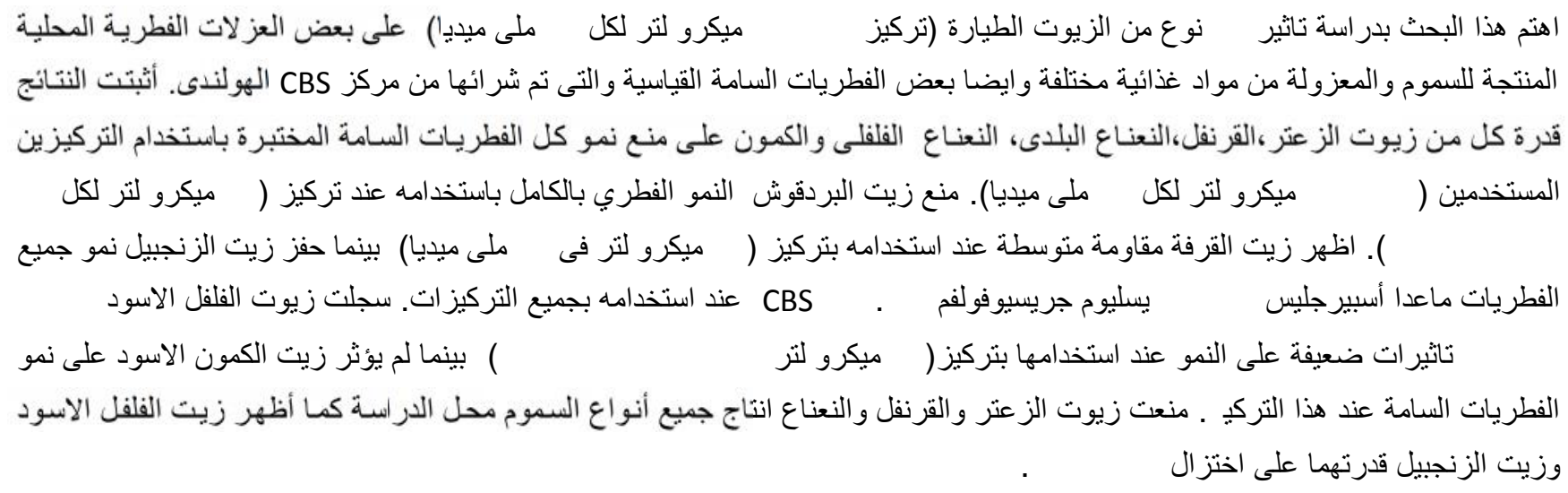

